\title{
Sym004-induced EGFR elimination is associated with profound anti- tumor activity in EGFRvIll patient-derived glioblastoma models
}

\author{
Stephen T. Keir ${ }^{1}\left[\right.$. Vidyalakshmi Chandramohan ${ }^{1} \cdot$ Carlee D. Hemphill $^{1} \cdot$ Michael M. Grandal ${ }^{2}$. \\ Maria Carlsen Melander ${ }^{2} \cdot$ Mikkel W. Pedersen $^{2} \cdot$ Ivan D. Horak $^{2} \cdot$ Michael Kragh $^{2} \cdot$ Annick Desjardins $^{1}$. \\ Henry S. Friedman ${ }^{1} \cdot$ Darell D. Bigner $^{1}$
}

Received: 23 January 2018 / Accepted: 14 March 2018 / Published online: 21 March 2018

(c) The Author(s) 2018

\begin{abstract}
Background Sym004 is a mixture of two monoclonal antibodies (mAbs), futuximab and modotuximab, targeting non-overlapping epitopes on the epidermal growth factor receptor (EGFR). Previous studies have shown that Sym004 is more efficient at inducing internalization and degradation of EGFR than individual components, which translates into superior cancer cell inhibition. We investigated whether Sym004 induces removal of EGFRvIII and if this removal translates into tumor growth inhibition in hard-to-treat glioblastomas (GBMs) harboring the mutated, constitutively active EGFR variant III (EGFRvIII). Methods To address this question, we tested the effect of Sym004 versus cetuximab in eight patient-derived GBM xenograft models expressing either wild-type EGFR (EGFRwt) and/or mutant EGFRvIII. All models were tested as both subcutaneous and orthotopic intracranial xenograft models.

Results In vitro studies demonstrated that Sym004 internalized and removed EGFRvIII more efficiently than mAbs, futuximab, modotuximab, and cetuximab. Removal of EGFRvIII by Sym004 translated into significant in vivo anti-tumor activity in all six EGFRvIII xenograft models. Furthermore, the anti-tumor activity of Sym004 in vivo was superior to that of its individual components, futuximab and modotuximab, suggesting a clear synergistic effect of the mAbs in the mixture. Conclusion These results demonstrate the broad activity of Sym004 in patient-derived EGFRvIII-expressing GBM xenograft models and provide a clear rationale for clinical evaluation of Sym004 in EGFRvIII-positive adult GBM patients.
\end{abstract}

Keywords GBM $\cdot$ Sym004 $\cdot$ EGFR $\cdot$ EGFRvIII $\cdot$ mAbs

\section{Importance of the study}

Glioblastoma (GBM) is an aggressive, highly invasive tumor with a median survival of 12-15 months. GBM tumors are associated with multiple genetic mutations and alterations, with approximately $50 \%$ having EGFR gene amplification.

Electronic supplementary material The online version of this article (https://doi.org/10.1007/s11060-018-2832-6) contains supplementary material, which is available to authorized users.

Stephen T. Keir

Keir0001@mc.duke.edu

1 Preston Robert Tisch Brain Tumor Center at Duke, Department of Neurosurgery, Duke University Medical Center, 3624 DUMC, Baker House, Durham, NC 27710, USA

2 Symphogen A/S, Pederstrupvej 93, 27500 Ballerup, Denmark
Approximately half of GBMs with EGFR gene amplification express a truncated EGFR (EGFRvIII) from a mutated gene lacking exons 2-7, which encode part of the extracellular ligand-binding domain. The truncated receptor displays a constitutive, ligand-independent tyrosine kinase activity and is associated with a more aggressive tumor phenotype than tumors expressing only EGFRwt. Therefore, new therapies active against EGFRvIII, such as Sym004, stand to improve treatment strategies for patients and warrant further exploration.

\section{Introduction}

Glioblastoma (GBM) is the most common, aggressive and subsequently lethal tumor of the central nervous system in adults [1, 2]. Although GBM therapy has improved in recent years, the prognosis for this disease still remains dismal, 
with median survival rates of $42.4 \%$ at 6 months and $17.7 \%$ at 1 year [2,3]. While numerous clinical trials seeking further improvements are underway, the current standard of care still involves maximum safe surgical resection or tumor debulking, followed by concomitant radiation and temozolomide (TMZ) therapy [4-6]. Despite encouraging advances in recent scientific research focusing on the genetic origins and molecular drivers of GBM, only modest improvements have been made in treating this fatal disease [7]. The poor prognosis associated with GBM results largely from a lack of understanding of its aggressive nature and insufficient therapeutic options [8]. Thus, there is a clear need for new treatments and targeted therapies for patients diagnosed with $\operatorname{GBM}[3,9,10]$.

Data from The Cancer Genome Atlas (TCGA) — National Cancer Institute project recently proposed various subtypes of GBM, where each subtype is characterized by its own distinct set of molecular properties and genetic aberrations [11]. TCGA data indicate that approximately $67 \%$ of all patients diagnosed with GBM have an aberrant amplification, deletion, or mutation of one or more receptor tyrosine kinase(s) [12]. The most frequent genetic change associated with GBM is amplification of the EGFR gene, which results in overexpression of the transmembrane tyrosine kinase receptor and occurs in approximately $57 \%$ of the GBM patient population $[12,13]$. In $50 \%$ of these cases, the EGFR amplification is accompanied by a gene rearrangement known as EGFRvIII [11, 13-15]. EGFRvIII is caused by an in-frame deletion of 801 base pairs of the coding sequence from exons $2-7$, resulting in a truncated gene encoding an aberrant receptor lacking two-thirds of the extracellular domain $[16,17]$. EGFRvIII is unable to bind EGFR ligands, but the receptor is constitutively active.

The over-expression of EGFR and/or its constitutively activated variant, EGFRvIII, is a major characteristic of GBM and is associated with aggressive, invasive, and therapeutic-resistant phenotypes [8]. The GBM cell line U87MG, which was retrovirally transfected with EGFR vIII (U87MG. EGFRvIII), showed significant growth advantage when grown as tumor xenografts, as well as advanced survivability under starvation serum conditions compared with the parental cell line [18, 19]. As EGFRvIII is often co-expressed with EGFRwt [20], both represent viable therapeutic targets for patients with GBM.

Sym004 is a 1:1 mixture of two recombinant humanmouse chimeric mAbs (futuximab and modotuximab) directed against non-overlapping EGFR epitopes. Preclinical studies with Sym004 have demonstrated activity against a variety of EGFR-expressing solid tumors [21-24]. Additionally, Sym004 has shown superior tumor growth inhibition in a range of xenograft models compared with other monoclonal anti-EGFR antibodies [21-24]. Sym004 is currently being evaluated in mCRC and GBM and has shown promising results in a phase I metastatic colorectal cancer trial with patients with chemotherapy-resistant/refractory tumors and acquired resistance to cetuximab and/or panitumumab [25]. The aim of the present study was to characterize the efficacy of Sym004 against a panel of EGFR positive patient-derived GBM xenografts with and without concomitant EGFRvIII expression.

\section{Materials and methods}

All patient-derived xenograft models used in this study were obtained from the Duke Brain Tumor Biorespository after receiving appropriate written consent by the Duke University Institutional Review Board. The final diagnosis of tumor samples was made and confirmed by the Duke Pathology Department. All models used have been cryopreserved and have extensive snap and viably frozen aliquots for testing and thawing. In general, all xenograft lines have been profiled with short tandem repeat using ABI Profiler and COfiler commercial reagent kits made by Applied Biosystems. These kits are used for DNA typing of all xenograft lines.

\section{Selection of xenografts}

EGFRwt and EGFRvIII statuses were determined by quantitative fluorescent activated cell sorter (QFACS) analysis. D08-0308MG and 43MG were found to express EGFRwt only. D10-0279MG and D10-0171MG were found to express only EGFRvIII, whereas D10-0319MG and D2159MG expressed both EGFRwt and EGFRvIII (Fig. 1). Xenograft models D270MG and D317MG were previously described in literature and not retested for this study, both co-express EGFRwt and EGFRvIII [26-28].

\section{Dissociation of xenografts}

Tissue from human biopsy-derived malignant glioma xenografts was obtained under sterile conditions from the Cancer Center Isolation Facility at Duke. Tumor tissue was processed and prepared for cell culture in a laminar flow hood under sterile conditions. The tumor material was finely minced and digested with $100 \mu \mathrm{g}$ of Liberase (Roche Indianapolis, IN). This mixture was stirred at $37^{\circ} \mathrm{C}$ for $10 \mathrm{~min}$, and a cell-rich supernatant was obtained. The cells were washed with a complete zinc option ( $\mathrm{ZO}$ ) medium, further treated with Ficoll-Hypaque to remove any red blood cells, and then 

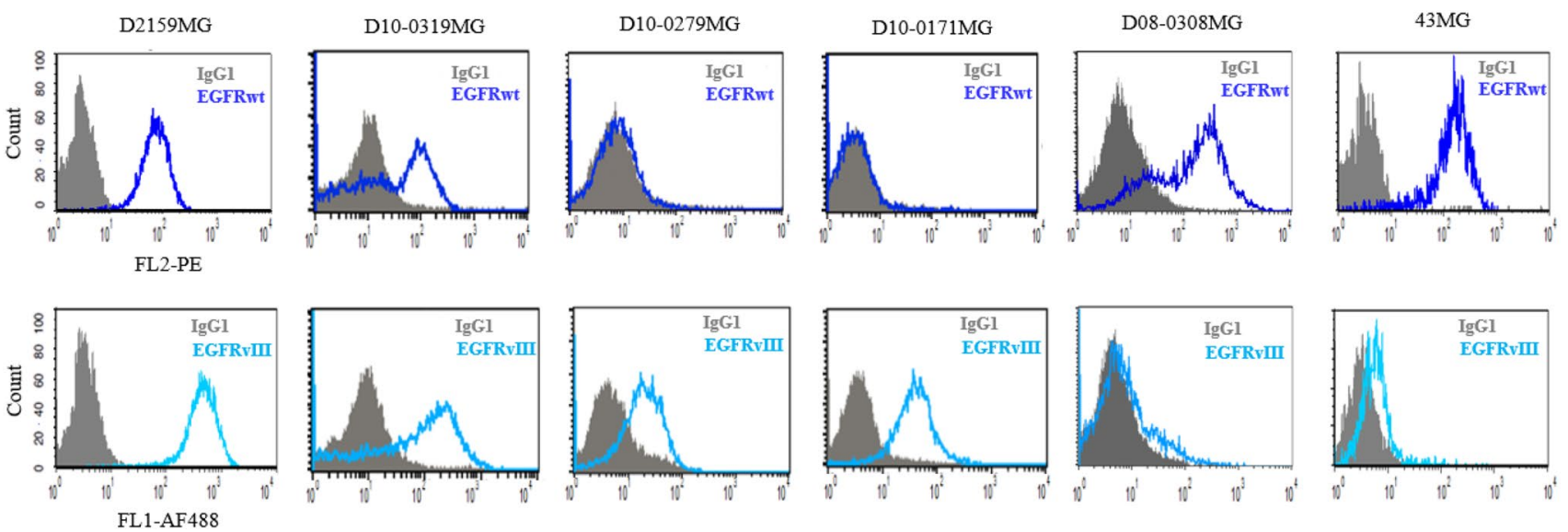

Fig. 1 EGFR Expression on GBM Xenograft Cells by QFACS. The number of EGFRwt and EGFRvIII receptors expressed on cells isolated from xenografts was determined by QFACS. EGFRwt expres-

washed once more in a $\mathrm{ZO}$ medium as described in previous studies [29, 30].

\section{Determination of receptor number by quantitative FACS analysis}

The number of EGFRwt and EGFRvIII receptors expressed on cells isolated from xenografts was determined by QFACS, using the Quantum Simply Cellular anti-Mouse IgG kit (Bangs Laboratories, Inc., Fishers, IN), as described in previous studies [29, 30]. To summarize, a cocktail of uniform-sized beads (one blank and four with varying capacities to bind to Mouse $\operatorname{IgG}$ ) was used for creating a standard curve and the cells were stained with $10 \mu \mathrm{g} / \mathrm{mL}$ of Mouse IgG2b-PE, EGFR1-PE (an EGFRwtspecific antibody), Mouse IgG1-AF488, or L8A4-AF488 (an EGFRvIII-specific antibody) for $45 \mathrm{~min}$ at $4{ }^{\circ} \mathrm{C}$. After washing, the beads and the cells were analyzed on a Becton Dickinson FACSCalibur instrument. Analysis of receptor density was performed by interpolation with the bead standard curves using QuickCal analysis software provided with the kit. The QFACS assays were performed on at least two different occasions on all brain tumor cells.

\section{Animals}

Male and female athymic mice (nu/nu genotype, Balb/c background, 6-8 weeks old) were used for all antitumor studies. The animals were maintained in an Allentown JAG75 PNC ventilated cage and rack system (Allentown, PA). All animal procedures conformed to the Institutional sion is featured in the upper panel and EGFRvIII expression in the lower panel. A shift to the right in the panels indicates presence of EGFRwt and/or EGFRvIII in xenografts used in this study

Animal Care and Use Committee's and the National Institute of Health's guidelines.

\section{Tumor xenografts and implantation}

Patient-derived human GBM xenografts maintained at The Preston Robert Tisch Brain Tumor Center were used for all studies.

In preparation for subcutaneous (s.c.) transplantation, s.c. xenografts passaged in athymic mice were excised from the host mice under sterile conditions in a laminar flow containment hood and placed into a modified tissue press. The resulting homogenate was then loaded into a repeating Hamilton syringe dispenser. The tumor homogenate was injected s.c. into the right flank of the athymic mouse at an inoculation volume of $50 \mu \mathrm{L}$ with a 19-gauge needle [31, 32].

For intracranial (i.c.) studies, s.c. xenografts passaged in athymic mice were excised from the host mice under sterile conditions in a laminar flow containment hood. The xenograft was minced and the cells were separated with a 60-mesh tissue cytosieve (BioWhittaker Inc., Walkersville, MD) into a ZO solution (Sigma-Aldrich, Allentown, PA), allowing for passage through a 25-gauge needle. After centrifugation, the supernatant was removed, and the cells were mixed 1:1 with methylcellulose. This mixture was then loaded into a repeating 250-/J Hamilton syringe (Hamilton, Co., Reno, NV) dispenser and injected i.c. at an inoculation volume of $10 \mu$ l. The i.c. injections were performed by placing a mouse into a stereotactic frame. A 1/2" midline skin incision was made. The bregma was located and the coordinates ( $2 \mathrm{~mm}$ lateral) were determined. A mounting holder on the frame supported the syringe containing the cells. A sterile 25-gauge needle attached to the syringe was 
introduced through the calvaria and into the brain at a depth of $4 \mathrm{~mm}$. The needle was then pulled back $0.5 \mathrm{~mm}$ to create a well for the homogenate. The xenograft homogenate was injected and after 1 min the syringe was pulled up and a small amount of bone wax was placed to occlude the hole. The mouse was removed from the frame and wound clips were used to close the skin [31, 32]. Lidocaine and Bupivicaine were used to control pain.

\section{Subcutaneous tumor measurement}

Subcutaneous tumors were measured twice weekly with hand-held vernier calipers (Scientific Products, McGraw, IL). Tumor volumes, V, were calculated with the following formula: $\mathrm{V}\left(\mathrm{mm}^{3}\right)=\left[(\text { width })^{2} \times(\right.$ length $\left.)\right] / 2$.

\section{Subcutaneous and intracranial xenograft therapy}

For the s.c. tumor studies, groups of 7-10 mice were stratified by tumor volume and were treated when the median tumor volumes were an average of $200 \mathrm{~mm}^{3}$. For i.c. tumor studies, groups of mice were randomized 3 days after i.c. tumor implantation, as previously described [33]. Groups of tumor-bearing animals received either Sym004, cetuximab, or TMZ and were compared to untreated controls.

\section{Antibodies and dosing}

Sym004, futuximab, and modotuximab were provided by Symphogen, Denmark. TMZ was purchased from Selleckchem (Houston, TX) and cetuximab was purchased from the Duke Inpatient Pharmacy. Sym004, futuximab, modotuximab and cetuximab were administered at a dose of $50 \mathrm{mg} /$ $\mathrm{kg}$ intraperitoneally (i.p.) twice weekly for 5 weeks (10 treatments total) and TMZ at a dose of $50 \mathrm{mg} / \mathrm{kg}$ i.p. for 5 consecutive days.

\section{Evaluation of subcutaneous xenograft response}

The response of the s.c. xenografts to treatment was assessed by a delay in tumor growth and differences in median values. Growth delay, expressed as $\mathrm{T}-\mathrm{C}$, was defined as the difference in days between the median time required for the tumors in the treated $(\mathrm{T})$ and control $(\mathrm{C})$ animals to reach a volume five times greater than that measured at the start of the treatment and/or reach a minimum volume of $>1000 \mathrm{~mm}^{3}$. Statistical analysis was performed using a SAS statistical analysis program. The Wilcoxon rank order test and Student $t$ test were used [31, 34-38].

\section{Evaluation of intracranial xenograft response}

The response of the i.c. xenografts to treatment was assessed by the percentage of increase in time to a specific neurologic endpoint (i.e., seizure activity, repetitive circling, 15\% decrease in weight or decrease in appetite) or to moribund status. Statistical analysis was performed using the Wilcoxon rank order test, as previously described [31, 32, 37-39]. All animals were observed twice daily for signs of distress or development of neurological symptoms, at which time they were removed from the study and euthanized.

\section{Receptor internalization}

After 1 day in medium with $2 \%$ serum, NR6M cells were incubated with $20 \mu \mathrm{g} / \mathrm{mL}$ Sym004, futuximab, modotuximab, cetuximab or the negative control $\mathrm{mAb}$ for the indicated periods. After treatment, cells were fixed, permeabilized, and incubated with anti-EGFR primary antibody (20ES04, Sheep, Fitzgerald). Subsequently, the nuclei, the primary antibody and treatment antibodies were stained with Hoechst, donkey anti-sheep IgG coupled to Alexa fluorophore 488 and goat anti-human IgG coupled to Alexa fluorophore 647, respectively. Images were acquired using Opera High Content Screening System with a 40× objective (PerkinElmer).

\section{Analysis of receptor modulation}

Lysates of cells treated with $20 \mu \mathrm{g} / \mathrm{mL}$ mAbs or Sym004 for 24 or $48 \mathrm{~h}$ were prepared as described elsewhere [40]. Samples for Simple Western analysis of total receptor levels were processed under standard conditions in a Sally Simple Western instrument (ProteinSimple). Rabbit primary antibodies against EGFRvIII from cell signaling technology (all diluted 1:50) were used. Statistically significant differences between untreated and treatment groups were calculated by one-way ANOVA with Bonferroni test post hoc correction.

\section{Results}

\section{Subcutaneous in vivo study}

Sym004 produced statistically significant $(\mathrm{P}<0.002)$ growth delays in seven of the eight subcutaneous GBM xenograft models tested (Fig. 2; Supplemental Table 1). The significant $\mathrm{T}-\mathrm{C}$ values for the s.c. studies ranged from 7.80 to 91 days and Sym004 outperformed cetuximab in the 2 EGFRvIIIexpressing models D10-0171MG and D10-0279MG and in 

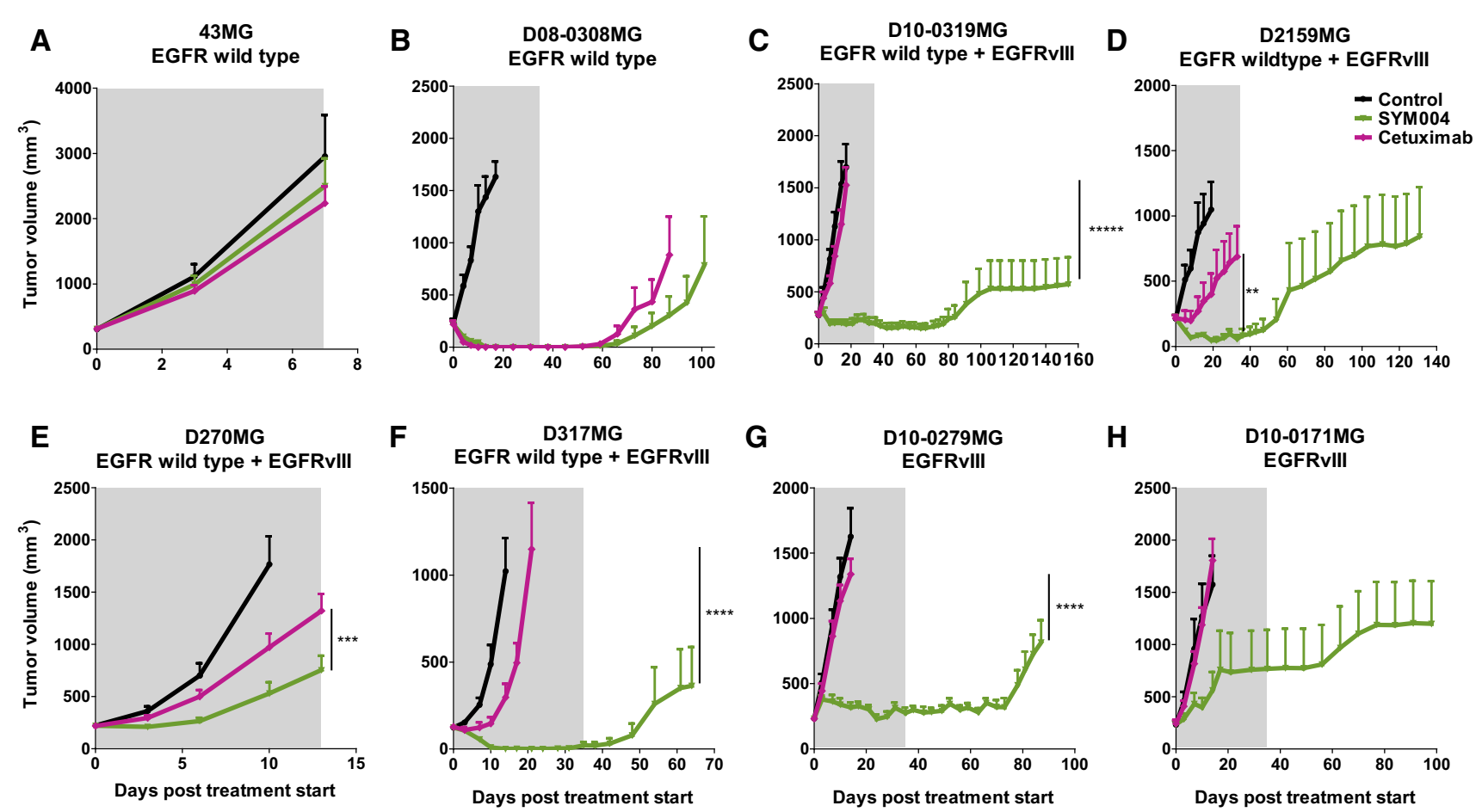

Fig. 2 Effect of Sym004 in Subcutaneous Glioblastoma Xenograft Tumor Models. Tumor growth curves for subcutanous GBM xenografts expressing (a, b) EGFRwt, (c-f) EGFRwt+EGFRvIII, or (g, h) EGFRvIII. Homogenate of eight patient-derived GBM xenografts passaged in athymic mice were injected s.c. in the right flank of $\mathrm{Balb} / \mathrm{c} \mathrm{nu} / \mathrm{nu}$ mice. At an average tumor size of $200 \mathrm{~mm}^{3}$, mice were randomized into four groups $(\mathrm{N}=7-10 /$ group $)$ and treatment initiated. Sym004 and cetuximab were administered at a dose of $50 \mathrm{mg} /$

xenografts expressing both EGFRwt and EGFRvIII (D100319MG, D2159MG, D317MG, and D270MG). The activity of cetuximab and Sym004 in the 2 EGFRwt xenograft lines (43MG and D08-0308MG) was similar.

\section{Intracranial in vivo study}

The increase in percentage survival for the Sym004 i.c. studies ranged from 39 to $305 \%$ and Sym004 significantly increased survival in seven of the eight models tested (Fig. 3; Supplemental Table 2). In the intracranial studies, Sym004 statistically outperformed cetuximab in the EGFRvIII alone expressing xenografts (D10-0171MG and D10-0279MG). In the models expressing both EGFRwt and EGFRvIII, Sym004 significantly outperformed cetuximab in D270MG, D-317MG, and D10-0319MG but not in D2159MG. As seen in the s.c. models, the activity of cetuximab and Sym004 in the two EGFRwt xenograft lines (43MG and D08-0308MG) was similar.

$\mathrm{kg}$ i.p. twice weekly for 5 weeks (10 treatments in total). The grey area denotes the treatment period. Control treated mice are shown in black, Sym004 in green and cetuximab in mauve. Two-way ANOVA with Bonferroni's multiple comparisons test was applied to compare tumor volumes at each time-point between Sym004 and cetuximab. Statistical analyses were performed using GraphPad Prism version 5.0 (GraphPad Software, Inc.). Data are presented as means \pm SEM. $* \mathrm{p}<0.05, * * * \mathrm{p}<0.001, * * * * \mathrm{p}<0.0001$

\section{Internalization and degradation of EGFRvIII by Sym004}

The ability of Sym004 to induce EGFRvIII internalization and degradation was investigated in the EGFRvIIIexpressing cell line NR6M. NR6M cells were incubated with Sym004, and the localization of EGFRvIII and Sym004 was detected with fluorophore-coupled secondary antibodies. Sym004 induced higher levels of EGFR internalization compared to individual antibodies (futuximab and modotuximab) and cetuximab (Fig. 4a, b) [41]. The fate of internalized receptors was investigated using quantitative Western blot analysis by Protein Simple. As shown in Fig. 4c, Sym004-treated NR6M cells had significantly lower levels of EGFRvIII compared to mAb-treated cells. After $48 \mathrm{~h}$, Sym004 decreased EGFRvIII levels to approximately onequarter of the levels in untreated cells, whereas mAbs had no impact or only slightly reduced EGFRvIII levels. 

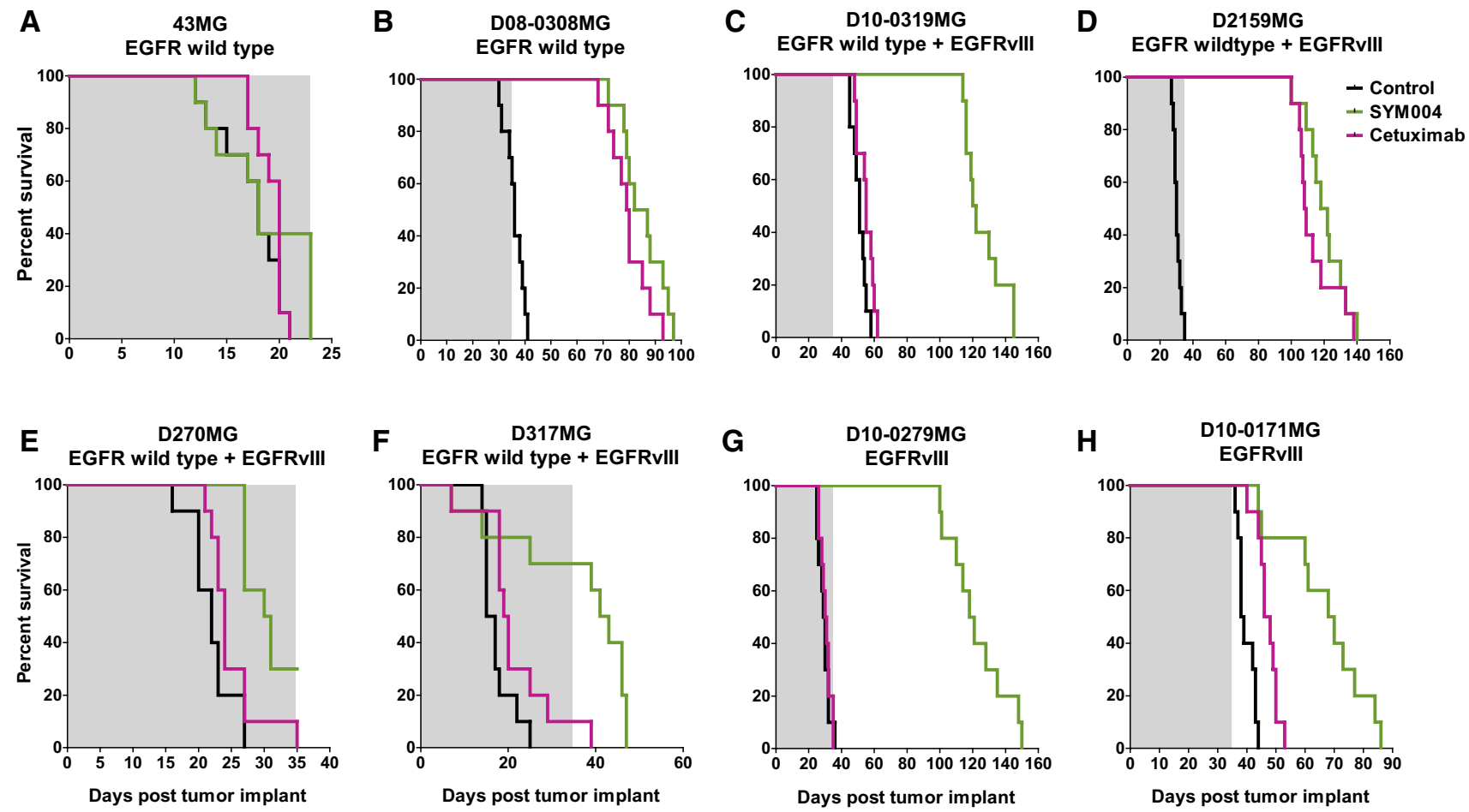

Fig. 3 Effect of Sym004 in orthotopic glioblastoma xenograft tumor models. Kaplan-Meier survival curves for mice bearing intracranial GBM xenografts expressing (a, b) EGFRwt, (c-f) EGFRwt+EGFRvIII, or (g, h) EGFRvIII. Homogenate of eight patient-derived GBM xenografts passaged in athymic Balb/c nu/

\section{Individual antibodies tested subcutaneously in vivo}

To test the hypothesis that the individual mAbs were working synergistically as an inhibitor of tumor growth, individual antibodies were tested in two s.c. models using D080308MG (EGFRwt) and D10-0171MG (EGFRvIII) and then compared to Sym004 (Fig. 5). In both the EGFRwt and EGFRvIII expressing xenograft models, Sym004 statistically outperformed the control and each individual antibody.

\section{Discussion/Conclusion}

GBM is the most common brain cancer in adults. Despite considerable attention from the research community, it remains a major therapeutic challenge with extremely poor clinical outcomes. Overexpression and amplification of EGFR, the most common genetic alternation in primary GBM, are associated with more aggressive and drug-resistant tumor phenotypes [42]. Of the primary GBMs that overexpress EGFR, up to $60 \%$ also express the tumor-specific variant, EGFRvIII [43]. Expression of EGFRvIII has been shown to lead to significant tumor and cell growth advantage in vivo and in vitro settings $[18,19]$. These pro-tumorigenic

nu mice were injected i.c. using a stereotactic frame. Sym004 and cetuximab were administered at a dose of $50 \mathrm{mg} / \mathrm{kg}$ i.p. twice weekly for 5 weeks (10 treatments in total, $\mathrm{N}=8-10$ /group). The grey area denotes the treatment period. Control treated mice are shown in black, Sym004 in green and cetuximab in mauve

properties of EGFRvIII can be linked to resistance to conventional therapies [13]. Unfortunately, most trials to date targeting EGFR and/or EGFRvIII have demonstrated little to no benefit [20, 44, 45]. Several clinical trials have evaluated the anti-EGFR mAb cetuximab. Though well tolerated by patients alone and in combination with other agents, only a small percentage of patients had an improvement in overall survival and experienced durable disease control. These studies indicate that patients with EGFR amplification had better responses than those without. Despite these encouraging results, there exists clear opportunities for continued development regarding therapies targeting the EGFR. Thus, the development of more efficacious cancer treatments and therapeutic options is extremely important for effective treatment in order to extend life and/or cure patients with GBM [46, 47].

The results from this study clearly demonstrate that Sym004 is active against patient-derived GBM xenografts expressing EGFR and/or EGFRvIII. In addition, it was shown that the two mAbs, futuximab, and modotuximab, comprising Sym004 work additively and even synergistically in the tested GBM models. The superior tumor growth inhibition induced by Sym004 in several of the s.c. GBM models and the prolonged survival upon Sym004 treatment 

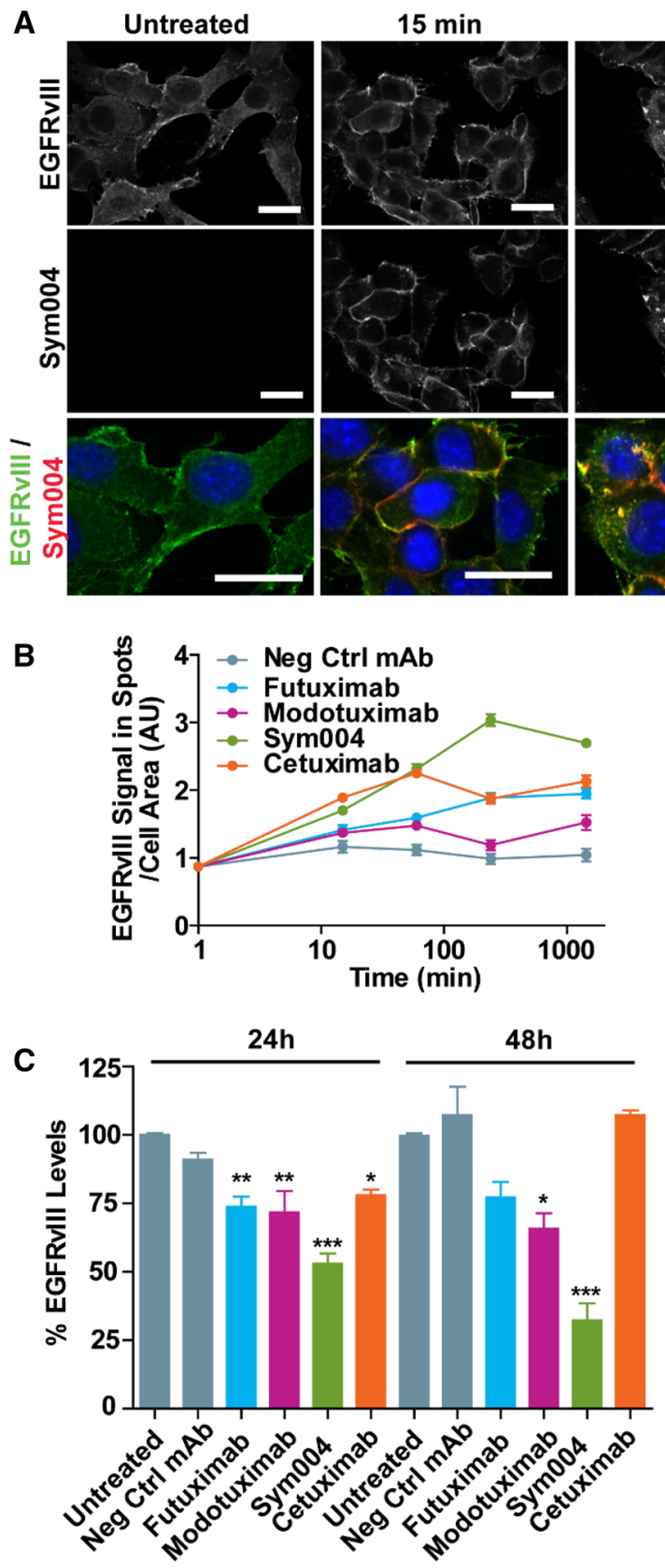

Fig. 4 Sym004 Internalizes and Degrades EGFRvIII. a NR6M cells were incubated with Sym004 for the indicated periods, and localization of EGFRvIII and Sym004 was detected with fluorophorecoupled secondary antibodies. The upper two panels show localization of EGFRvIII and Sym004, respectively. The lower panels show magnified composite images. Green, red, and blue indicate localization of EGFRvIII, Sym004, and nuclei, respectively. b Quantification of EGFRvIII and antibodies detected in spots after antibody treat-
$1 \mathrm{~h}$
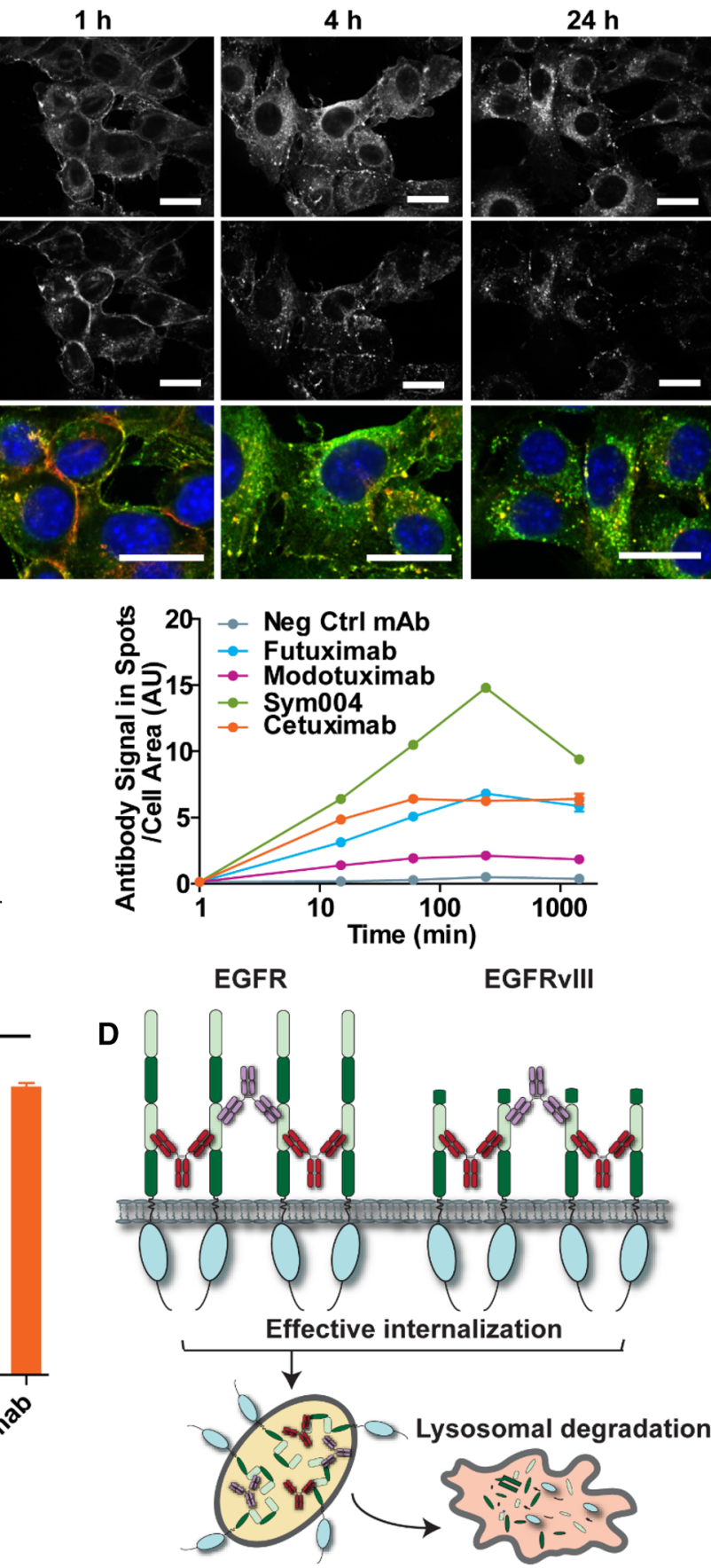

Endosome

ment for the indicated periods. c The cell line NR6M was treated with negative control $\mathrm{mAb}$ (Neg $\mathrm{Ctrl} \mathrm{mAb}$ ), futuximab, modotuximab, or Sym004 for 24 or $48 \mathrm{~h}$, followed by harvest of cell lysates and detection of EGFRvIII by Western blot. Data are represented as means \pm SEM. Statistically significant differences from untreated controls are indicated by asterisks $* \mathrm{p}<0.05, * * \mathrm{p}<0.01, * * * \mathrm{p}<0.001$. d Proposed model of internalization and degradation of EGFR and EGFRvIII by Sym004 


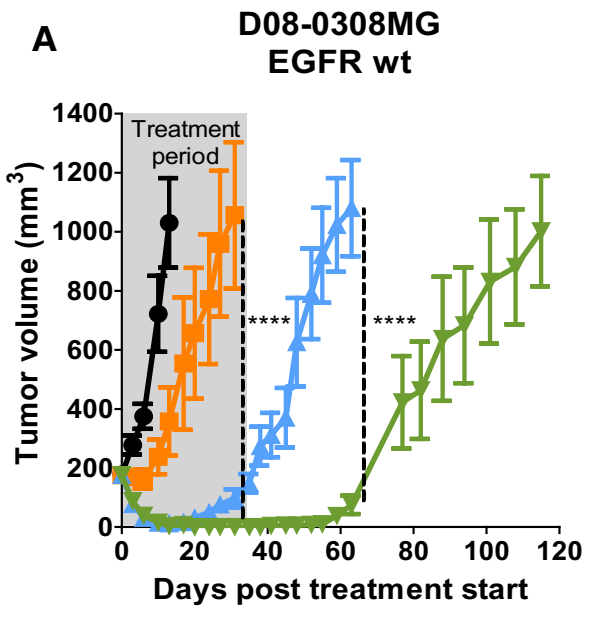

Fig. 5 Effect of Sym004, and Its Components Futuximab and Modotuximab, in Subcutaneous Glioblastoma Xenograft Tumor Models. Homogenate of a EGFRwt (D08-0308MG) and b EGFRvIII (D100171MG) patient-derived GBM xenografts passaged in athymic mice were injected s.c. in the right flank of Balb/c nu/nu mice. At an average tumor size of $200 \mathrm{~mm}^{3}$, mice were randomized into four groups $(\mathrm{N}=10 /$ group) and treatment initiated. Sym004, futuximab, and modotuximab were administered at a dose of $50 \mathrm{mg} / \mathrm{kg}$ i.p. twice weekly for 5 weeks (10 treatments in total). The grey area denotes the

in the i.c. models compared to cetuximab, demonstrate the potential of Sym004 for treatment of GBM.

EGFRvIII is constitutively active, unable to bind EGFR ligands and escapes downregulation due to inefficient internalization and/or transport to lysosomes. A potential explanation for the superior activity of Sym004 compared to the individual mAbs and cetuximab could be the ability of Sym004 to induce EGFR, cross-linking, internalization and degradation [22].

The two antibodies futuximab and modotuximab bind non-overlapping epitopes on domain III of EGFR, a domain that is intact in EGFRvIII. It was therefore expected that $\mathrm{Sym} 004$ were able to induce internalization and degradation of EGFRvIII similar to EGFRwt. Indeed, we were able to demonstrate that Sym004 induces efficient internalization and degradation of EGFRvIII thereby effectively shutting down oncogenic signaling. Sym004 also engages secondary effector functions such as antibody dependent cellular cytoxicity (ADCC) and/or complement dependent cytoxicity (CDC), which contributes to the drug candidates overall anti-tumor activity [21].

Sym004 thus has the unique potential to effectively target both EGFRwt and EGFRvIII in GBM providing a clear rationale for evaluating Sym004 in patients with GBM. An ongoing phase 2 trial in patients with GBM will assess the

\section{B D10-0171MG EGFRvIII}

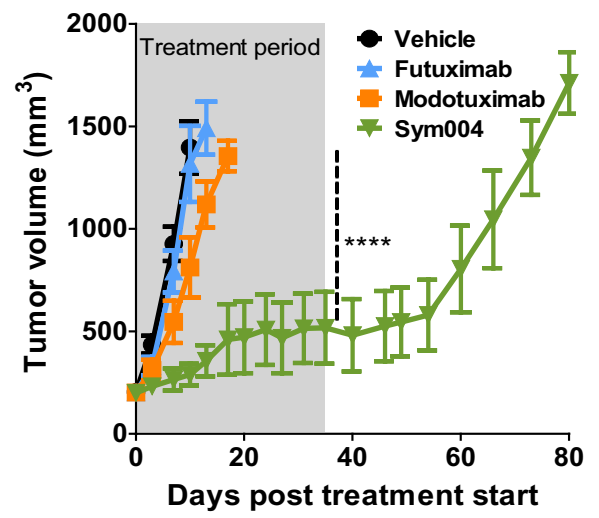

treatment period. Control treated mice are shown in black, Sym004 in green, futuximab in blue, and modotuximab in orange. Two-way ANOVA with Bonferroni's multiple comparisons test was applied to compare tumor volumes at each time-point between Sym004 and futuximab and modotuximab respectively. Statistical analyses were performed using GraphPad Prism version 5.0 (GraphPad Software, Inc.). Data are presented as means \pm SEM. $* \mathrm{p}<0.05, * * * \mathrm{p}<0.001$, $* * * * \mathrm{p}<0.0001$

safety and efficacy of Sym004 in patients with progression after primary therapy.

Funding Symphogen A/S; Merck KGaA and the Tisch Preclinical Therapy Screening Program.

\section{Compliance with ethical standards}

Conflict of interest The authors declared that they have no conflicts of interest.

Ethical approval All procedures involving animals conformed the Institutional Animal Care and Use Committee's and the National Institute of Health's guidelines.

Open Access This article is distributed under the terms of the Creative Commons Attribution 4.0 International License (http://creativeco mmons.org/licenses/by/4.0/), which permits unrestricted use, distribution, and reproduction in any medium, provided you give appropriate credit to the original author(s) and the source, provide a link to the Creative Commons license, and indicate if changes were made.

\section{References}

1. Ohgaki H, Kleihues P (2005) Population-based studies on incidence, survival rates, and genetic alterations in astrocytic and oligodendroglial gliomas. J Neuropathol Exp Neurol 64:479-489 
2. Wen PY, Kesari S (2008) Malignant gliomas in adults. New Eng J Med 359(5):492-507

3. Delgado-Lopez PD, Corrales-Garcia EM (2016) Survival in glioblastoma: a review on the impact of treatment modalities. Clin Transl Oncol 18(11):1062-1071

4. Stupp R, Mason WP, van den Bent MJ et al (2005) Radiotherapy plus concomitant and adjuvant temozolomide for glioblastoma. New Eng J Med 352(10):987-996

5. Stupp R, van den Bent MJ, Hegi ME (2005) Optimal role of temozolomide in the treatment of malignant gliomas. Curr Neurol Neurosci Rep 5(3):198-206

6. Stupp R, Weber DC (2005) The role of radio- and chemotherapy in glioblastoma. Onkologie 28:315-317

7. Turtoi A, Blomme A, Bianchi E et al (2014) Accessibilome of human glioblastoma: collagen-VI-alpha-1 is a new target and a marker of poor outcome. J Proteome Res 13(12):5660-5669

8. Lo HW (2010) EGFR-targeted therapy in malignant glioma: novel aspects and mechanisms of drug resistance. Curr Mol Pharmacol $3(1): 37-52$

9. Schernberg A, Marabelle A, Massard C et al (2016) What's next in glioblastoma treatment: tumor-targeted or immune-targeted therapies? Bull Cancer 103(5):484-498

10. Jue TR, McDonald KL (2016) The challenges associated with molecular targeted therapies for glioblastoma. J Neurooncol 127(3):427-434

11. Verhaak RG, Hoadley KA, Purdom E et al (2010) Integrated genomic analysis identifies clinically relevant subtypes of glioblastoma characterized by abnormalities in PDGFRA, IDH1, EGFR, and NF1. Cancer Cell 17(1):98-110

12. Wheeler DL, Huang S, Kruser TJ et al (2008) Mechanisms of acquired resistance to cetuximab: role of HER (ErbB) family members. Oncogene 27(28):3944-3956

13. Heimberger AB, Suki D, Yang D, Shi W, Aldape K (2005) The natural history of EGFR and EGFRvIII in glioblastoma patients. $\mathrm{J}$ Transl Med 3:38

14. Wheeler DL, Iida M, Kruser TJ et al (2009) Epidermal growth factor receptor cooperates with Src family kinases in acquired resistance to cetuximab. Cancer Biol Ther 8(8):696-703

15. Pelloski CE, Ballman KV, Furth AF et al (2007) Epidermal growth factor receptor variant III status defines clinically distinct subtypes of glioblastoma. J Clin Oncol 25(16):2288-2294

16. Zadeh G, Bhat KP, Aldape K (2013) EGFR and EGFRvIII in glioblastoma: partners in crime. Cancer Cell 24(4):403-404

17. Huang PH, Xu AM, White FM (2009) Oncogenic EGFR signaling networks in glioma. Sci Signal 2(87):re6

18. Nagane M, Coufal F, Lin H, Bogler O, Cavenee WK, Huang HJ (1996) A common mutant epidermal growth factor receptor confers enhanced tumorigenicity on human glioblastoma cells by increasing proliferation and reducing apoptosis. Cancer Res 56(21):5079-5086

19. Nishikawa R, Ji XD, Harmon RC et al (1994) A mutant epidermal growth factor receptor common in human glioma confers enhanced tumorigenicity. Proc Natl Acad Sci USA 91(16):7727-7731

20. Padfield E, Ellis HP, Kurian KM (2015) Current therapeutic advances targeting EGFR and EGFRvIII in glioblastoma. Front Oncol 5:5

21. Koefoed K, Steinaa L, Soderberg JN et al (2011) Rational identification of an optimal antibody mixture for targeting the epidermal growth factor receptor. MAbs 3(6):584-595

22. Pedersen MW, Jacobsen HJ, Koefoed K et al (2010) Sym004: a novel synergistic anti-epidermal growth factor receptor antibody mixture with superior anticancer efficacy. Cancer Res 70(2):588-597

23. Sanchez-Martin FJ, Bellosillo B, Gelabert M et al (2016) The firstin-class anti-EGFR antibody mixture Sym004 overcomes cetuximab-resistance mediated by EGFR extracellular domain mutations in colorectal cancer. Clin Cancer Res 22(13):3260-3270
24. Iida M, Brand TM, Starr MM et al (2013) Sym004, a novel EGFR antibody mixture, can overcome acquired resistance to cetuximab. Neoplasia 15(10): 1196 - 206

25. Dienstmann R, Patnaik A, Garcia-Carbonero R et al (2015) Safety and activity of the first-in-class Sym004 anti-EGFR antibody mixture in patients with refractory colorectal cancer. Cancer Discov 5(6):598-609

26. Bao X, Pastan I, Bigner DD, Chandramohan V (2016) EGFR/ EGFRvIII-targeted immunotoxin therapy for the treatment of glioblastomas via convection-enhanced delivery. Recept Clin Investig 3(4): 1430

27. Wong AJ, Ruppert JM, Bigner SH et al (1992) Structural alterations of the epidermal growth factor receptor gene in human gliomas. Proc Natl Acad Sci USA 89(7):2965-2969

28. Zhu H, Cao X, Ali-Osman F, Keir S, Lo H-W (2010) EGFR and EGFRvIII interact with PUMA to inhibit mitochondrial translocalization of PUMA and PUMA-mediated apoptosis independent of EGFR kinase activity. Cancer Lett 294(1):101-110

29. Chandramohan V, Bao X, Keir ST et al (2013) Construction of an immunotoxin, D2C7-(scdsFv)-PE38KDEL, targeting EGFRwt and EGFRvIII for brain tumor therapy. Clin Cancer Res 19(17):4717-4727

30. Wikstrand CJ, McLendon RE, Friedman AH, Bigner DD (1997) Cell surface localization and density of the tumor-associated variant of the epidermal growth factor receptor, EGFRvIII. Cancer Res 57(18):4130-4140

31. Friedman HS, Colvin OM, Skapek SX et al (1988) Experimental chemotherapy of human medulloblastoma cell lines and transplantable xenografts with bifunctional alkylating agents. Cancer Res 48(15):4189-4195

32. Carlson BL, Pokorny JL, Schroeder MA, Sarkaria JN (2011) Establishment, maintenance and in vitro and in vivo applications of primary human glioblastoma multiforme (GBM) xenograft models for translational biology studies and drug discovery. Curr Protoc Pharmacol 52(14):1-14

33. Pollack IF, Bredel M, Erff M, Hamilton AD, Sebti SM (1999) Inhibition of Ras and related guanosine triphosphate-dependent proteins as a therapeutic strategy for blocking malignant glioma growth: II-preclinical studies in a nude mouse model. Neurosurgery 45(5):12081214 discussion $14-5$

34. Chandramohan V, Bao X, Kato Kaneko M et al (2013) Recombinant anti-podoplanin (NZ-1) immunotoxin for the treatment of malignant brain tumors. Int J Cancer 132(10):2339-2348

35. Friedman HS, Houghton PJ, Schold SC, Keir S, Bigner DD (1994) Activity of 9-dimethylaminomethyl-10-hydroxycamptothecin against pediatric and adult central nervous system tumor xenografts. Cancer Chemother Pharmocol 34(2):171-174

36. Keir ST, Friedman HS, Reardon DA, Bigner DD, Gray LA (2013) Mibefradil, a novel therapy for glioblastoma multiforme: cell cycle synchronization and interlaced therapy in a murine model. J Neurooncol 111(2):97-102

37. Keir ST, Dewhirst MW, Kirkpatrick JP, Bigner DD, Batinic-Haberle I (2011) Cellular redox modulator, ortho Mn(III) meso-tetrakis(N-nhexylpyridinium-2-yl)porphyrin, $\mathrm{MnTnHex}-2-\mathrm{PyP}(5+)$ in the treatment of brain tumors. Anticancer Agents Med Chem 11(2):202-212

38. Keir ST, Hausheer F, Lawless AA, Bigner DD, Friedman HS (2001) Therapeutic activity of 7-[(2-trimethylsilyl)ethyl)]-20 (S)-camptothecin against central nervous system tumor-derived xenografts in athymic mice. Cancer Chemother Pharmocol 48(1):83-87

39. Gehan EA (1965) A generalized two-sample Wilcoxon test for doubly censored data. Biometrika 52(3):650-653

40. Jacobsen HJ, Poulsen TT, Dahlman A et al (2015) Pan-HER, an antibody mixture simultaneously targeting EGFR, HER2, and HER3, effectively overcomes tumor heterogeneity and plasticity. Clin Cancer Res 21(18):4110-4122 
41. Batra SK, Castelino-Prabhu S, Wikstrand CJ, Zhu X, Humphrey PA, Friedman HS et al (1995) Epidermal growth factor ligand-independent, unregulated, cell-transforming potential of a naturally occurring human mutant EGFRvIII gene. Cell Growth Differ 6:1251-1259

42. Ekstrand AJ, James CD, Cavenee WK et al (1991) Genes for epidermal growth factor receptor alpha, transforming growth factor alpha, and epidermal growth factor and their expression in human gliomas in vivo. Cancer Res 51:2164-2172

43. Sugawa N, Ekstrand AJ, James CD, Collins VP (1990) Identical splicing of aberrant epidermal growth factor receptor transcripts from amplified rearranged genes in human glioblastomas. Proc Natl Acad Sci USA 87(21):8602-8606
44. Rich JN, Reardon DA, Peery T et al (2004) Phase II trial of gefitinib in recurrent glioblastoma. J Clin Oncol 22(1):133-142

45. De Witt Hamer PC (2010) Small molecule kinase inhibitors in glioblastoma: a systematic review of clinical studies. Neuro Oncol 12(3):304-316

46. Neyns B, Sadones J, Joosens E et al (2009) Stratitfied phase II trial of cetuximab in patients with recurrent high-grade glioma. Ann Oncol 20(9):1596-1603

47. Hasselbalch B, Lassen U, Hansen S et al (2010) Cetuximab, bevacizumab, and irinotecan for patients with primary glioblastoma and progression after radiation therapy and temozolomide: a phase II trial. Neuro Oncol 12(5):508-516 